\section{Kidney \\ Blood Pressure Research}

Kidney Blood Press Res 2015;40:244-257

DOI: 10.1159/000368500

Published online: May 08, 2015

Accepted: March 31, 2015

\title{
Molecular Patterns of Subclinical and Clinical Rejection of Kidney Allograft: Quantity Matters
}

\author{
Mariana Wohlfahrtova ${ }^{a, b}$ Irena Tycova ${ }^{b}$ Eva Honsovac $^{c}$ Alena Lodererova ${ }^{3}$ \\ Ondrej Viklickya,b
}

aDepartment of Nephrology, Transplant Center; ${ }^{\mathrm{b}}$ Transplant Laboratory; ${ }^{~} \mathrm{Clinical}$ and Transplant Pathology Department, Institute for Clinical and Experimental Medicine, Prague, Czech Republic

\section{Key Words}

Kidney transplantation - PCR - Protocol biopsy - Subclinical rejection - Transcriptome • Prostaglandin receptor

\begin{abstract}
Background/Aims: Subclinical rejection diagnosed from protocol biopsies is thought to be a risk factor of long- term allograft dysfunction. The reason why in some patients subclinical rejection does not represent risk for progression is not fully understood. Methods: The intragraft expression of 376 target genes involved in chemokine defense, apoptosis, inflammation, tolerance and TGF- $\beta$ signalling pathways was measured using quantitative real-

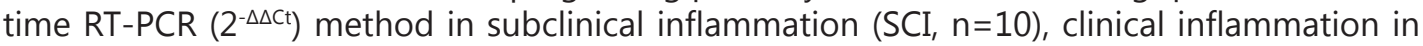
acute $T$-cell mediated rejection $(\mathrm{CI}, \mathrm{n}=10)$ and no rejection samples $(\mathrm{n}=9)$. Results: Clinical inflammation group showed a increased expression of genes for chemotaxis mediating cytokines (CCL1, CCL17, CCL24, CCL25, CCL26), cytokine receptors (CCR1, CCRL2, IL1RAPL2, CXCR5), proinflammatory cytokines (IL12A, LTA), inflammatory mediator (PTAFR), complement protein $C 3$, executioner protein of apoptosis (CASP7), growth factor (TGFA), colony stimulating factor (CSF-2), proteins involved in dendritic cells differentiation and interaction (CD209, $\angle A M P 3)$, regulation of immune response (LILRB2, LILBRB4). The quantitative difference in transcripts signature between $\mathrm{SCI}$ and $\mathrm{CI}$ is consistent with stronger proinflammatory setting of CI. Prostaglandin E2 receptor gene expression was independently associated with lower risk of further graft function deterioration (OR 0.11, CI 0.01-0.78, p<0.0001). Conclusion: Subclinical acute kidney inflammation has transcriptional profile of immune injury of lower extend compared to clinical acute inflammation.
\end{abstract}




\section{Kidney Blood Pressure Research}

Kidney Blood Press Res 2015;40:244-257

\begin{tabular}{l|l}
\hline DOI: $10.1159 / 000368500$ & (C) 2015 S. Karger AG, Basel
\end{tabular}

Published online: May 08, 2015

www.karger.com/kbr

\section{Introduction}

Despite improvements in firstyear of kidney allograft survival, chronic rejection remains to be the main reason for late kidney allograft loss [1]. In the development of chronic rejection, a subclinical alloimmune injury may play an important role [2]. The incidence of subclinical acute rejection may vary and depends on time post transplantation, immunosuppressive regimen and previous sensitization [1, 3-6]. Therapy of subclinical rejection remains to be a subject of debate. While some authors referred benefits of subclinical rejection treatment [7-10], others did not $[11,12]$.

The explanation why subclinical rejection is not accompanied by functional deterioration in some patients remains to be speculative $[9,11,13]$. A different phenotype and cytokine activity of infiltrating cells [14] along with quantitative difference in transcriptional activity have been suggested to be responsible for the absence of graft function deterioration [15].

Although subclinical rejection of kidney allograft might have negative impact on allograft survival and some investigators have attempted to account for differences between SCI and clinical acute rejection (CI) on molecular level [16-19], no detailed differences have yet been fully elucidated. Transcriptome alterations detected by molecular analysis might even precede minor subclinical infiltrates in histology of protocol biopsies [20]. Several attempts have been made to predict short- and long-term graft outcome by molecular analyses of protocol biopsies [21,22]. The identification of such molecular predictors and thereby grafts at risk seems to be crucial for future biomarker-driven therapy in transplantation.

We hypothesized that molecular phenotype evaluation might provide novel insight into the pathogenesis and clinical relevance of subclinical rejection of kidney allograft. Thus we evaluated the molecular phenotype of subclinical inflammation (SCI) from 3-month protocol biopsies and clinical inflammation (CI) from case biopsies and searched for potential molecular predictors of graft function deterioration.

\section{Patients and Methods}

\section{Study Design and Patients}

To analyze molecular phenotype of distinct clinical course of subclinical and clinical rejection, we identified study patients among kidney transplant recipients from deceased donor transplanted in 06/2005-06/2008 whose part of biopsy specimens was archived for transcriptome analyses. Rigorous sample selection was based on morphologic finding according to Banff 2005 classification. Patients with BK nephropathy have been excluded from the study.

Subclinical inflammation group (SCI) $(n=10)$ involved patients with finding of borderline changes or acute T-cell mediated rejection (TCMR) in protocol biopsy at month 3 in the absence of clinical dysfunction (increase in S- $\mathrm{Cr}<10 \%$, no proteinuria) and with no history of prior rejection [9]. Clinical inflammation group (CI) $(\mathrm{n}=10)$ involved kidney recipients with borderline changes or acute T- cell mediated rejection in early case biopsy within 3 months after kidney transplantation which was performed due to clinical signs of graft function deterioration. Control group ( $\mathrm{n}=9$ ) consisted of patients with normal histological findings in protocol biopsies performed in stable renal allografts at 3 months after transplantation. Acute rejection was treated according to KDIGO clinical practice guidelines including treatment of borderline changes and subclinical rejection [23]. All patients were followed for 24 months. The Ethics Committee of the Institute for Clinical and Experimental Medicine approved the study protocol and all patients signed informed consent to participate in the study (IRB approval number G08-08-10). This study was conducted in accordance with Helsinki Declaration.

\section{Immunosuppressive Treatment}

Immunosuppression consisted of tacrolimus (TAC), mycophenolate mofetil (MMF), steroids in all patients but two from CI group who received maintenance therapy based on cyclosporine A. These two patients did not experience graft function deterioration. Two patients (1 SCI, 1 control group) were given induction treatment with basiliximab and one with rabbit antithymocytary globulin (SCI). As one of the major factors that may influence the development of SCI is suboptimal immunosuppression we carefully 


\section{Kidney \\ Blood Pressure Research}

Kidney Blood Press Res 2015;40:244-257

\begin{tabular}{|l|l|}
\hline DOI: $10.1159 / 000368500$ & (c 2015 S. Karger AG, Basel \\
\hline
\end{tabular}

Published online: May 08, 2015

www.karger.com/kbr

Wohlfahrtova/Tycova/Honsova/Lodererova/Viklicky: Molecular Pattern of Subclinical Rejection

looked for adequacy of immunosuppressive treatment which was adequate and comparable across the studied groups.

\section{Protocol Biopsy}

Protocol kidney graft biopsy was performed in accordance with our centre practice at 3 months after transplantation; early case biopsy was performed within 3 months postTx. Biopsies were obtained under ultrasound guidance (Toshiba, Power Vision 6000) using a 14-gauge Tru-Cut needle (Somatex ${ }^{\oplus}$; Medical Technologies GmbH, Germany). A small portion ( $2 \mathrm{~mm}$ ) of the cortical zone of the biopsy specimen was immediately placed in the RNA later Stabilization Reagent (Qiagen) and stored at $-20^{\circ} \mathrm{C}$ or $-80^{\circ} \mathrm{C}$ until RNA extraction.

RNA Extraction and cDNA Synthesis [24].

The entire procedure, ranging from RNA extraction to cDNA synthesis, is in detail described elsewhere

Real-time RT-PCR Analysis Using TaqMan Low Density Arrays (TLDA)

Gene expression profiling was performed using a custom-made Taqman ${ }^{\circledR}$ low density array (TLDA, Applied Biosystems, CA, USA) to analyze 376 candidate genes known to have implications in immune response (chemokine defence, apoptosis, inflammation, tolerance and TGF $\beta$ signaling). Quantitative realtime RT-PCR assay based on TLDA technology was performed as described elsewhere [25]. Real- time RTqPCR data were quantified using the SDS 2.4 software package (Applied Biosystems, Foster City, CA, USA).

\section{Expression Data Analysis}

Relative gene expression values were generated from TLDA analysis using the comparative $2^{-\Delta \Delta C t}$ method for relative quantification, which is implemented in the Applied Biosystems Relative Quantification (RQ) Manager Software v1.2.1 (Applied Biosystems, Foster City, CA, USA) [26]. For calculating the RQ of target genes in transplanted kidney, renal tissue from protocol biopsy with normal histological finding, not included in control group, was designated as calibrator. Using the $2^{-\Delta \Delta C t}$ method, data are presented as the fold change in gene expression normalized to an endogenous reference gene and relative to the calibrator sample. All qRT-PCR data were rigorously inspected before included into analysis. All evaluated samples expressed more than $60 \%$ of target genes.

\section{Immunohistochemistry}

Immunohistochemical detection of prostaglandin E receptor 2 /PTGER 2/ was performed on $4 \mu \mathrm{m}$ thick paraffin sections of early case and 3 month protocol renal graft biopsies using a two-step indirect method. The slides were deparaffinized in xylene and rehydrated in graded ethanol. Heat-induced epitope retrieval in the buffer was used to improve the reactivity of antibody. Endogenous peroxidase was blocked by $0.3 \% \mathrm{H}_{2} \mathrm{O}_{2}$ in $70 \%$ methanol for 30 minutes. Primary antibody was applied (PTGER 2, origin: rabbit, clone: polyclonal, Acris, dilution: 100x, application: 30 min RT, buffer: citrate, $\mathrm{pH}$ 6). Detection of antibody was performed using Simple Stain MAX PO (MULTI) Universal Immuno-peroxidase Polymer, Anti-Mouse and -Rabbit -Histofine (Nichirei, Japan). Finally, specimens were stained with Dako AEC + High Sensitivity (Dako, Glostrup, Denmark) for 10 min and were counterstained with Mayer's hematoxilin.

\section{Statistical Analysis}

Student $t$ test and the analysis of variance were applied to the variables following a normal distribution. Based on gene expression data distribution, we performed nonparametric testing and data were expressed as median and interquartile range. Group comparison for median RQ values was determined using KruskalWallis test. Post-hoc-inter-group comparisons were made using Mann-Whitney test with adjustment for multiple comparisons. Significance was defined as a two sided p value $<0.05$. Association between continuous variables (RQ gene expressions, allograft function) and ordinal variables (Banff scores) was assessed as Spearman correlation. The genes considered to be differentially expressed had not to fulfil only statistical but also biological significance, when minimal cut-off for differential expression was set to 2 -fold. Multivariate logistic regression analysis was conducted to search for significant associations of possible predicting factors with respect to the outcome variable (progression of renal dysfunction). Due to low patient number, logistic regression modelling was restricted to 5 significant genes from univariate analysis of the highest biological and statistical relevance. The area under the ROC curve (c-statistic) derived from this 


\section{Kidney \\ Blood Pressure Research}

Wohlfahrtova/Tycova/Honsova/Lodererova/Viklicky: Molecular Pattern of Subclinical Rejection

Table 1. Patients'demographics and clinical characteritics according to studied groups

\begin{tabular}{|c|c|c|c|c|}
\hline & $\begin{array}{c}\text { control group } \\
(n=9)\end{array}$ & $\begin{array}{c}\mathrm{SCI} \\
(\mathrm{n}=10)\end{array}$ & $\begin{array}{c}\mathrm{CI} \\
(\mathrm{n}=10)\end{array}$ & $P$ \\
\hline Recipient age (yr) & $45 \pm 13.26$ & $52.8 \pm 5.63$ & $54.7 \pm 10.586$ & 0.183 \\
\hline Recipient gender ( $\%$ male) & $5(55.6)$ & $5(50)$ & $9(90)$ & 0.128 \\
\hline Mean \% PRA at transplantation & $6.22 \pm 10.32$ & $15.2 \pm 31.74$ & $15 \pm 31.34$ & 0.721 \\
\hline Previous transplant & 0 & 0 & 0 & 1 \\
\hline HLA mismatch & $3.89 \pm 1.05$ & $2.9 \pm 0.99$ & $4 \pm 0.82$ & 0.031 \\
\hline Cold ischemia time (hr) & $17.61 \pm 2.86$ & $17.44 \pm 4.09$ & $17.04 \pm 2.06$ & 0.942 \\
\hline Dialysis vintage $(\mathrm{d})$ & $669(307-1330)$ & $642(422-1474)$ & $462(314-813)$ & 0.535 \\
\hline Grafts with delayed graft function (\%) & $2(22.2 \%)$ & $5(50 \%)$ & $1(10 \%)$ & 0.123 \\
\hline Graft function development (d) & $1.67 \pm 3.39$ & $4.6 \pm 4.93$ & $0.9 \pm 2.85$ & 0.088 \\
\hline Biopsy (postTx d) & $99 \pm 9.55$ & $94.5 \pm 4.17$ & $28.7 \pm 31.41$ & 0.001 \\
\hline Donor age (yr) & $41.56 \pm 12.27$ & $44.3 \pm 15.84$ & $46.43 \pm 12.88$ & 0.899 \\
\hline \multicolumn{4}{|l|}{$\begin{array}{l}\text { Maintenance immunosuppressive } \\
\text { regimen at biopsy }\end{array}$} & \multirow[t]{3}{*}{ NS } \\
\hline Tacrolimus, MMF, Steroids & $9(100 \%)$ & $10(100 \%)$ & $8(80 \%)$ & \\
\hline Cyclosporine A, MMF, Steroids & 0 & 0 & $2(20 \%)$ & \\
\hline \multicolumn{5}{|l|}{ Serum creatinine $(\mu \mathrm{mol} / \mathrm{L})$} \\
\hline Baseline (at biopsy) & $113 \pm 28.95$ & $133.7 \pm 40.19$ & $244 \pm 96.61$ & 0,0003 \\
\hline yr 1 & $114.56 \pm 33.00$ & $233.97 \pm 214.3$ & $211.33 \pm 147.17$ & 0.229 \\
\hline yr 2 & $150.66 \pm 108.68$ & $225.42 \pm 199.23$ & $227.08 \pm 140.82$ & 0.491 \\
\hline \multicolumn{5}{|l|}{$\begin{array}{l}\text { GFR estimated with MDRD } \\
\left(\mathrm{ml} / \mathrm{sec} / 1.73 \mathrm{~m}^{2}\right)\end{array}$} \\
\hline Baseline (at biopsy) & $1.05 \pm 0.22$ & $0.78 \pm 0.27$ & $0.49 \pm 0.15$ & $<0,0001$ \\
\hline yr 1 & $1.06 \pm 0.34$ & $0.66 \pm 0.36$ & $0.61 \pm 0.30$ & 0.12 \\
\hline yr 2 & $0.89 \pm 0.29$ & $0.64 \pm 0.38$ & $0.55 \pm 0.26$ & 0.069 \\
\hline \multicolumn{5}{|l|}{ Proteinuria $(\mathrm{g} / \mathrm{d})$} \\
\hline Baseline (at biopsy) & $0.13 \pm 0.12$ & $0.38 \pm 0.46$ & $0.67 \pm 0.48$ & 0.24 \\
\hline yr 1 & $0.066 \pm 0.073$ & $0.787 \pm 0.779$ & $0.254 \pm 0.342$ & 0.011 \\
\hline yr 2 & $0.194 \pm 0.156$ & $0.821 \pm 1.304$ & $0.319 \pm 0.357$ & 0.22 \\
\hline
\end{tabular}

logistic regression analysis reflects the model's discriminative power. Unsupervised hierarchical clustering that grouped together genes with similar expression patterns was performed with MultiExperiment Viewer 4.6.0 (TM4, Boston, MA, USA).

\section{Results}

\section{Patients' Characteristics and Outcome}

In total, 29 renal allograft biopsies were studied. Out of these samples, ten samples were derived from protocol biopsies with subclinical rejection including borderline changes (SCI), ten samples from early case biopsies with finding of clinical rejection and borderline changes (CI). Nine patients with normal histological finding in protocol biopsies served as control group. Transplantation- associated risk factors such as cold ischemia time, PRA level, etc. were not significantly different between studied groups (Table 1) except for total HLA mismatch and the day of biopsy taken resulting from the study setting. Among the patients studied, 8 patients developed delayed graft function (DGF) defined as the need for dialysis during the first week after transplantation [27], with 5 of these patients being from SCI group.

\section{Histological Evaluation of Studied Biopsies}

Severity of rejection grades was comparable across rejection groups. No difference in inflammation rate was observed between SCI and CI group. No active antibody mediated rejection was included into our study. The histological lesions scores show comparable chronic graft injury across the studied groups (Table 2).

Differential gene expression in biopsies derived from SCI and CI

To detect subtle alterations associated with the absence of graft function deterioration in SCI, mRNA expression profile of kidney allografts was compared. This analysis identified 23 


\section{Kidney Blood Pressure Research}

\begin{tabular}{l|l}
\hline Kidney Blood Press Res 2015;40:244-257 \\
\hline DOI: $10.1159 / 000368500$ & $\begin{array}{l}\text { C } 2015 \text { S. Karger AG, Basel } \\
\text { www.karger.com/kbr }\end{array}$ \\
\hline Published online: May 08, 2015 & .
\end{tabular}

Wohlfahrtova/Tycova/Honsova/Lodererova/Viklicky: Molecular Pattern of Subclinical Rejection

Table 2. Histological evaluation of kidney biopsies according to Banff 05 classification

\begin{tabular}{lccccc}
\hline & $\begin{array}{c}\mathrm{SCI} \\
(\mathrm{n}=10)\end{array}$ & $\begin{array}{c}\mathrm{CI} \\
(\mathrm{n}=10)\end{array}$ & $\begin{array}{c}\text { control } \\
(\mathrm{n}=9)\end{array}$ & $\begin{array}{c}\mathrm{p} \text { - Value } \\
(\text { SCI vs. CI })\end{array}$ & $\mathrm{p}^{\dagger}$-Value* \\
\hline borderline/gradeI/gradeII & $(4 / 3 / 3)$ & $(3 / 2 / 5)$ & & & \\
i- score & $1(0-2)$ & $2(0.75-2)$ & $0(0-0)$ & 0.162 & 0.001 \\
t- score & $1(0-2)$ & $1(0.5-2)$ & $0(0-0)$ & 0.76 & 0.002 \\
ci- score & $0(0-1)$ & $0(0-0.25)$ & $0(0-0)$ & 0.342 & 0.105 \\
ct- score & $1(0-1)$ & $1(0.75-1)$ & $1(0-1)$ & 0.423 & 0.440 \\
v- score & $0(0-1)$ & $0(0-0.25)$ & $0(0-0)$ & 0.473 & 0.093 \\
cv- score & $1(0.5-2)$ & $1(0-1)$ & $0(0-1)$ & 0.140 & 0.113 \\
g- score & $0(0-0)$ & $0(0-0.25)$ & $0(0-0)$ & 0.146 & 0.139 \\
cg- score & $0(0-0)$ & $0(0-0)$ & $0(0-0)$ & 1 & 1 \\
mm- score & $0(0-0)$ & $0(0-0)$ & $0(0-0)$ & 0.317 & 0.401 \\
ah- score & $0(0-1)$ & $1(0-1)$ & $1(0-1.5)$ & 0.426 & 0.548 \\
\hline
\end{tabular}

Dara are expressed as median (interquartile range).

$\dagger$ according to the Mann- Whitney U test, no corrected for multiple comparisons

* according to Kruskal Wallis test

Table 3. The genes showing different expressions between SCI and CI group

\begin{tabular}{|c|c|c|c|c|c|c|}
\hline target gene & SCI & $\mathrm{CI}$ & control & $\begin{array}{c}P^{\#} \\
\text { (CI vs. SCI) }\end{array}$ & $\begin{array}{c}n \text {-fold } \\
\text { (CI vs.SCI) }\end{array}$ & $P^{*}$ \\
\hline $\mathrm{C} 3$ & $1.61(0-6.04)$ & $11.65(2.16-49.63)$ & $2.02(0-2.76)$ & 0.015 & 7.24 & .021 \\
\hline CASP7 & $0.47(0.22-0.88)$ & $1.27(0.89-2.3)$ & $1.13(0.59-1.17)$ & 0.006 & 2.70 & .014 \\
\hline CCL17 & $0.92(0.26-3.87)$ & $10.38(0.89-48.69)$ & $0.16(0-0.71)$ & 0.034 & 11.28 & .004 \\
\hline CCL1 & $0(0-0)$ & $3.05(0-9.08)$ & $0(0-0)$ & 0.031 & N/A & .017 \\
\hline CCL24 & $0(0-0)$ & $2.4(0.57-6.42)$ & $0(0-0)$ & 0.013 & $\mathrm{~N} / \mathrm{A}$ & .004 \\
\hline CCL25 & $1(1-1)$ & $13.41(1-75.06)$ & $1(1-1)$ & 0.007 & 13.41 & .001 \\
\hline CCL26 & $0.28(0-1.98)$ & $3.51(0.84-27.89)$ & $0.07(0-1.02)$ & 0.012 & 12.54 & .009 \\
\hline CCR1 & $0.52(0-1.35)$ & $2.02(0.59-3.52)$ & $0(0-0)$ & 0.049 & 3.88 & .017 \\
\hline CCRL2 & $0.61(0-1.84)$ & $3.33(1.09-7.74)$ & $0.5(0-1-99)$ & 0.018 & 5.46 & .036 \\
\hline CD209 & $0.25(0-0.7)$ & $1.02(0.51-3.09)$ & $0(0-0)$ & 0.032 & 4.08 & .001 \\
\hline CSF2 & $0.39(0-8.09)$ & $7.83(1.08-47.03)$ & $0(0-0)$ & 0.040 & 20.08 & .002 \\
\hline CXCR5 & $0.02(0-0.8)$ & $0.37(0.15-4.25)$ & $0(0-0)$ & 0.049 & 18.50 & .005 \\
\hline FOXP3 & $0.07(0-0-77)$ & $0.78(0.19-4.1)$ & $0(0-0)$ & 0.033 & 11.14 & .013 \\
\hline HPGD & $0.83(0.32-1.27)$ & $0.2(0.12-0.48)$ & $0.9(0.71-2.13)$ & 0.013 & 4.15 & .001 \\
\hline IL12A & $0(0-0)$ & $0.84(0.54-2.82)$ & $0(0-0)$ & 0.004 & N/A & .020 \\
\hline IL1RAPL2 & $1(1-1)$ & $9.24(1-50.55)$ & $1(1-1)$ & 0.039 & 9.24 & .004 \\
\hline KNG1 & $1.43(0.79-2.5)$ & $0.49(0.1-0.87)$ & $2.06(1.43-3.77)$ & 0.002 & 2.92 & .001 \\
\hline LAMP3 & $0(0-0)$ & $3.67(0.89-9.01)$ & $0(0-0)$ & 0.008 & $\mathrm{~N} / \mathrm{A}$ & .003 \\
\hline LILRB2 & $0.65(0-4.96)$ & $9.49(1.37-20.17)$ & $1(0.09-1.95)$ & 0.045 & 14.60 & .049 \\
\hline LILRB4 & $0.36(0.15-1.1)$ & $2.12(0.45-6.73)$ & $0.16(0-0.44)$ & 0.034 & 5.89 & .008 \\
\hline LTA & $0.01(0-0.2)$ & $0.45(0.12-1.24)$ & $0(0-0)$ & 0.024 & 45.00 & .027 \\
\hline PTAFR & $1.11(0.48-1.92)$ & $4.04(1.06-10.5)$ & $0.4(0.1-1.42)$ & 0.041 & 3.64 & .021 \\
\hline TGFA & $0.35(0-1.13)$ & $1.18(0.89-3.47)$ & $0(0-0)$ & 0.028 & 3.37 & .033 \\
\hline
\end{tabular}

Only differentially expressed genes fulfilling statistical and biological significance criteria are shown (\# according to Mann Whitney U-test). For complex view, results of Kruskal Wallis test* for all studied groups are shown.

genes with different expression between SCI and CI (Table 3). CI group showed a significant upregulation of genes for chemotaxis mediating cytokines (CCL1, CCL17, CCL24, CCL25, CCL26), cytokine receptors (CCR1, CCRL2, IL1RAPL2, CXCR5), proinflammatory cytokines (IL12A, LTA), inflammatory mediator (PTAFR), complement protein C3, executioner protein of apoptosis (CASP7), growth factor (TGFA), colony stimulating factor (CSF-2), proteins involved in dendritic cells differentiation and interaction (CD209, LAMP3) and in regulation of immune response (LILRB2, LILBRB4) compared to SCI. We observed not only quantitative difference between two rejection groups, but also trend of increasing expression of proinflammatory genes rising from control through subclinical to clinical acute rejection group.

Furthermore, we were able to detect higher expression of Treg-specific cell marker, 15-hydroxyprostaglandin dehydrogenase (HPGD) and kinogen-1 (KNG1) $(\mathrm{p}<0.01)$ in control and subclinical samples compared to clinical inflammation. An additional gene of interest was FoxP3, regulator of the development and function of regulatory $\mathrm{T}$ cells [28], which 


\section{Kidney Blood Pressure Research}

showed lower gene expression in SCI biopsies compared to CI $(\mathrm{p}<0.01)$.

Associations of molecular phenotype with histological scores for inflammation

In order to determine whether different gene expression profile of subclinical and clinical acute rejection was not related to severe grades of cellular rejection in CI, we correlated RQ of significant genes with the histological inflammation scores. This analysis revealed mostly positive correlation of some of evaluated genes with Banff score for tubulitis and interstitial inflammation but no correlation with glomerulitis, intimal arteritis was found (data not shown). We neither found association of these 23 differently expressed genes with DGF, future graft rejection nor function deterioration within 2 years posttransplantation (data not shown).

\section{Unsupervised hierarchical clustering}

Unsupervised hierarchical cluster analysis applied to all dataset did not produce a dendrogram with well-defined cluster groups. Performing unsupervised hierarchical clustering restricted to 23 differentially expressed genes revealed a significant up-regulation of proinflammatory genes in clinical inflammation group. Furthermore, it correctly classified all $10 \mathrm{CI}$ samples to separated cluster (Figure 1). Out of four SCI samples clustered together with CI samples, two belonged to patients with consequent graft function deterioration and failed within two years and one belonged to patient with subclinical finding of severe TCMR grade II.

\section{Graft function deterioration}

Despite notably worse baseline renal function in CI group, no differences between SCI and CI were found at the end of year 2 (Table 1). However, some patients showed renal function deterioration during the follow-up. Four patients from SCI group and three patients from CI group experienced further renal function worsening caused by repeated rejection episodes. Additionally, one patient from control group experienced an episode of clinical acute TCMR within the first year (day 186) due to noncompliance. Hereinafter, he experienced renal function worsening due to repeated urinary tract infections and lost his graft due to abscessed pyelonephritis 25 months after kidney transplantation. The demographics and clinical characteristics of progressors and nonprogressors are summarized in Table 4.

\section{Patient and graft survival}

In total, two patients from SCI and one patient from CI group lost their graft due to repetitious rejection episodes (month 10,11, 4, respectively). No graft loss due to patient death was observed. 


\section{Kidney Blood Pressure Research}

\begin{tabular}{l|l}
\hline Kidney Blood Press Res 2015;40:244-257 \\
\hline DOI: $10.1159 / 000368500$ & $\begin{array}{l}\text { C } 2015 \text { S. Karger AG, Basel } \\
\text { www.karger.com/kbr }\end{array}$ \\
\hline Published online: May 08, 2015 & Pat
\end{tabular}

Wohlfahrtova/Tycova/Honsova/Lodererova/Viklicky: Molecular Pattern of Subclinical Rejection

Table 4. Patients' demographics and clinical characteristics according to the groups based on graft function deterioration

\begin{tabular}{lccc}
\hline & $\begin{array}{c}\text { Nonprogressors } \\
(\mathrm{n}=13)\end{array}$ & $\begin{array}{c}\text { Progressors } \\
(\mathrm{n}=7)\end{array}$ & $P$ \\
\hline Recipient age (yr) & $54.7 \pm 9.6$ & $52 \pm 5.4$ & 0.504 \\
Recipient gender (\% male) & $10(76.9)$ & $3(42.9)$ & 0.174 \\
Mean \% PRA at transplantation & $14.3 \pm 28.5$ & $16.6 \pm 36.9$ & 0.88 \\
Previous transplant & 0 & 0 & 0 \\
HLA mismatch & $3.54 \pm 0.88$ & $3.29 \pm 1.38$ & 0.621 \\
Cold ischemia time (hr) & $16.15 \pm 1.57$ & $17.86 \pm 4.88$ & 0.400 \\
Dialysis vintage (d) & $606.8 \pm 331.3$ & $999 \pm 856.9$ & 0.155 \\
Delayed graft function (\%) & $2(15.38)$ & $3(42.85)$ & 0.290 \\
Graft function development (d) & $2.08 \pm 4.01$ & $4.00 \pm 5.00$ & 0.384 \\
Biopsy (postTx d) & $49.69 \pm 43.43$ & $83.71 \pm 21.55$ & 0.087 \\
Donor age (yr) & $53.92 \pm 6.37$ & $42.71 \pm 19.99$ & 0.194 \\
Maintenance immunosuppressive regimens & & & 0.521 \\
at biopsy (\%) & $11(84.61)$ & $7(100 \%)$ & \\
$\quad$ Tacrolimus, MMF, Steroids & $2(15.39)$ & 0 & \\
Cyclosporine A, MMF, Steroids & & & \\
Serum creatinine ( $\mu$ mol/L) & $202.1 \pm 87.7$ & $164.3 \pm 100.6$ & 0.154 \\
$\quad$ Baseline (at biopsy) & $148.7 \pm 29.0$ & $370.2 \pm 224.5$ & 0.057 \\
$\quad$ yr 2 & $-53.3 \pm 81.2$ & $205.9 \pm 209.4$ & 0.0004 \\
\hline Cr (yr 2-mo 3) & & & \\
\hline Data are expressed as mean \pm SD or n (\%). & & &
\end{tabular}

Differential expression of selected candidate genes is indicative of graft function deterioration

Next, we investigated whether evaluated transcriptome showed different phenotype in allografts suffering from later graft function deterioration. 7 patients that experienced worsening of graft function during 2 year follow-up were marked as progressors. Patients from control group were not involved into analysis when looking for potential predictors of renal function deterioration as all patients except for the aforementioned noncompliant one have stable renal function and because of different pre- and post- biopsy clinical course free of rejection and antirejection treatment.

Biopsy samples of progressors and nonprogressors were compared in order to identify genes of possible predictive value. 55 genes discriminating between progressors and nonprogressors were identified (Table 5). All genes fulfilled biological and statistical selection criteria. To evaluate whether these candidate genes are indicative of graft function deterioration, we performed multiple logistic regression analysis including five most biologically and statistically relevant genes (BNIP3L, PTGER2, CD1B, NFKBIE, MAPK 14) known from the univariate analysis to be differentially expressed in progressors. Multiple logistic regression analysis revealed that prostaglandin E2 receptor (PTGER2) gene expression level was associated with lower risk of graft function deterioration in studied biopsies (OR 0.11, 95\% CI $0.01-0.78$, Nagelkerke $\mathrm{R}^{2}=0.743, \mathrm{p}<0.0001$ ). The receiver operator characteristic analysis confirmed low PTGER2 gene expression to be associated with progression of renal dysfunction $(\mathrm{p}<0.0001$, AUC 0.878 , Figure 2$)$.

The role of PTGER2 in further graft function deterioration was also confirmed at the protein level. Immunohistochemical (IHC) evaluation of kidney graft biopsy specimens showed higher intensity of PTGER2 staining in tubular epithelial cells in patients without progression of graft dysfunction within the follow-up (Figure 3).

\section{Discussion}

The explanation why subclinical inflammation is not accompanied by graft function deterioration varies. This study attempted to find possible explanation for silent course of 


\section{Kidney \\ Blood Pressure Research}

\section{Kidney Blood Press Res 2015;40:244-257}

\begin{tabular}{l|l}
\hline DOI: $10.1159 / 000368500$ & (C) 2015 S. Karger AG, Basel
\end{tabular}

Published online: May 08, 2015

www.karger.com/kbr

251

Wohlfahrtova/Tycova/Honsova/Lodererova/Viklicky: Molecular Pattern of Subclinical

Rejection

Table 5. The genes showing different expressions between progressors and nonprogressors

\begin{tabular}{|c|c|c|c|c|}
\hline & Nonprogressors & Progressors & n-fold & $P$ \\
\hline BNIP3L & $1.50(1.04-1.92)$ & $0.76(0.69-0.96)$ & 2.0 & 0.002 \\
\hline PTGER2 & $3.70(1.71-15.16)$ & $0.59(0-1.37)$ & 6.3 & 0.002 \\
\hline CD1B & $6.15(1.14-12.65)$ & $0.09(0-1.09)$ & 72.4 & 0.004 \\
\hline MAPK14 & $1.44(0.98-2.16)$ & $0.57(0-0.89)$ & 2.5 & 0.006 \\
\hline NFKBIE & $1.44(0.52-3.51)$ & $0.29(0-0.56)$ & 4.9 & 0.006 \\
\hline CCL17 & $7.18(1.06-38.29)$ & $0.30(0.14-1.1)$ & 23.8 & 0.008 \\
\hline IL1R1 & $1.34(0.84-5.13)$ & $0.45(0.18 .0 .61)$ & 3.0 & 0.008 \\
\hline NFKB1 & $2.64(1.46-4.21)$ & $0.91(0.82-1.16)$ & 2.9 & 0.008 \\
\hline PLA2G4C & $1.26(0.86-2.59)$ & $0.47(0.31-0.95)$ & 2.7 & 0.008 \\
\hline LTB4R & $2.71(0.76-5.36)$ & $0.68(0.35-0.98)$ & 4.0 & 0.01 \\
\hline CXCL6 & $13.45(2.24-40.23)$ & $1.16(0.68-5.73)$ & 11.6 & 0.013 \\
\hline SOCS5 & $2.88(1.13-5.2)$ & $0.70(0.53-1.14)$ & 4.1 & 0.013 \\
\hline CD1C & $2.11(0.55-4.46)$ & $0.21(0.16-0.5)$ & 9.9 & 0.016 \\
\hline HRH1 & $1.85(0.57-5.62)$ & $0.37(0.35-1.13)$ & 5.0 & 0.016 \\
\hline IL2 & $1.21(0.05-4.01)$ & $0.00(0-0.19)$ & 12110.0 & 0.016 \\
\hline SELP & $1.23(0.55-1.71)$ & $0.22(0.1-0.54)$ & 5.7 & 0.016 \\
\hline CCL19 & $3.08(0.33-8.6)$ & $0.15(0.1-1.38)$ & 21.1 & 0.019 \\
\hline CCL26 & $2.39(0.48-24.16)$ & $0.13(0-0.91)$ & 18.1 & 0.019 \\
\hline SPARC & $1.29(0.74-1.49)$ & $0.56(0.52-0.79)$ & 2.3 & 0.019 \\
\hline FCGR2B & $2.72(0.73-12.04)$ & $0.68(0.18-0.87)$ & 4.0 & 0.024 \\
\hline IL10 & $4.74(0.73-12.78)$ & $0.00(0-2.47)$ & 47400.0 & 0.026 \\
\hline CD209 & $0.86(0.58-2.46)$ & $0.00(0-0.91)$ & 8605.0 & 0.027 \\
\hline $\mathrm{CD} 28$ & $2.48(0.51-7.01)$ & $0.10(0-0.75)$ & 24.6 & 0.027 \\
\hline IL8 & $6.26(2.2-17.18)$ & $1.53(0-3.1)$ & 4.1 & 0.028 \\
\hline XCL1 & $1.41(0.87-8.6)$ & $0.20(0-0.62)$ & 7.2 & 0.028 \\
\hline AK3L1 & $0.25(0.04-0.57)$ & $0.62(0.43-1.3)$ & $2.46^{*}$ & 0.029 \\
\hline CCL11 & $9.15(4.38-25.08)$ & $1.82(0.7-2.8)$ & 5.0 & 0.029 \\
\hline TNFSF13B & $9.67(1.92-21.63)$ & $2.14(0.53-5.16)$ & 4.5 & 0.029 \\
\hline VCAM1 & $4.77(2.25-7.74)$ & 1.27 (1.06-2.99) & 3.8 & 0.029 \\
\hline CCL20 & $101.88(21.46-429.74)$ & $13.82(6.72-21.71)$ & 7.4 & 0.03 \\
\hline ITGAM & $6.02(2.16-16.52)$ & $0.84(0-2.62)$ & 7.2 & 0.03 \\
\hline LTA & $0.35(0.06-1.58)$ & $0.00(0-0.13)$ & 3520.0 & 0.033 \\
\hline TGFB3 & $1.00(0.3-2.52)$ & $0.18(0-0.5)$ & 5.6 & 0.033 \\
\hline NLRP1 & $1.62(0.35-2.91)$ & $0.18(0-1.3)$ & 8.8 & 0.034 \\
\hline CD14 & $3.08(1.12-6.83)$ & $0.90(0.37-2.1)$ & 3.4 & 0.036 \\
\hline CFLAR & $2.23(1.13-3.7)$ & $1.09(0.79-1.41)$ & 2.1 & 0.036 \\
\hline CXCR4 & $4.07(0.95-8.13)$ & $0.99(0.36-1.92)$ & 4.1 & 0.036 \\
\hline GZMK & $2.74(0.78-9.52)$ & $0.51(0.1-1.48)$ & 5.3 & 0.036 \\
\hline IKBKB & $1.51(0.64-2.51)$ & $0.66(0.5-0.74)$ & 2.3 & 0.036 \\
\hline IL18 & $1.76(0.64-3.19)$ & $0.52(0.35-1.47)$ & 3.4 & 0.036 \\
\hline IL2RG & $2.37(0.67-4.42)$ & $0.69(0.09-1.4)$ & 3.4 & 0.036 \\
\hline TLR2 & $4.09(1.74-10.31)$ & $1.17(0.88-2.78)$ & 3.5 & 0.036 \\
\hline PTGDR & $2.51(0.81-6.3)$ & $0.31(0.15-0.78)$ & 8.0 & 0.037 \\
\hline CXCL2 & $4.69(2.15-31.92)$ & $1.14(0.6-2.54)$ & 4.1 & 0.039 \\
\hline TGFBR1 & $1.59(0.63-3.32)$ & $0.30(0-0.82)$ & 5.4 & 0.039 \\
\hline CD80 & $205.00(72.83-326.9)$ & $45.57(12.7-72.23)$ & 4.5 & 0.042 \\
\hline BCL2L11 & $1.77(0.89-4.26)$ & $0.70(0-1.21)$ & 2.5 & 0.043 \\
\hline BID & $3.97(1-13.7)$ & $0.89(0.55-1.31)$ & 4.5 & 0.043 \\
\hline CD40LG & $0.99(0.3-2.03)$ & $0.23(0.11-0.77)$ & 4.4 & 0.043 \\
\hline CKLF & $5.26(2.75-16.27)$ & $1.67(1.29-5.86)$ & 3.2 & 0.043 \\
\hline LTA4H & $1.78(0.97-3.1)$ & $0.90(0.72-1.59)$ & 2.0 & 0.043 \\
\hline TBK1 & $2.40(0.92-3.66)$ & $0.95(0.69-1.56)$ & 2.5 & 0.043 \\
\hline LAMP3 & $2.79(0.42-5.79)$ & $0.06(0-0.82)$ & 46.8 & 0.045 \\
\hline CD3E & $2.18(0.51-3.35)$ & $0.46(0.11-1.05)$ & 4.8 & 0.047 \\
\hline TP53 & $1.84(1.48-6.29)$ & $0.93(0.77-1.85)$ & 2.0 & 0.047 \\
\hline
\end{tabular}




\section{Kidney \\ Blood Pressure Research}

Kidney Blood Press Res 2015;40:244-257

\begin{tabular}{l|l}
\hline DOI: $10.1159 / 000368500$ & (C) 2015 S. Karger AG, Basel
\end{tabular}

Published online: May 08, 2015

www.karger.com/kbr

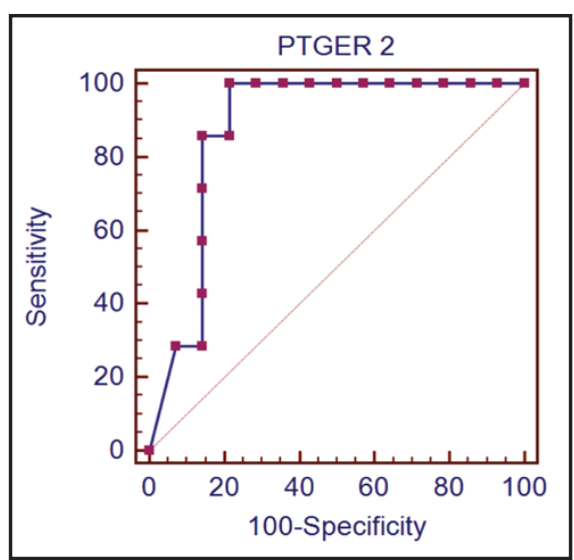

Fig. 2. Prediction of graft function deterioration. Receiver operator characteristic (ROC) curve for graft function deterioration using multiple regression analysis of 5 the most significant gene variables indicates receiver operator characteristic area under the curve (AUC); sensitivity defines true-positive results and 100- specificity false- positive results. The area under the ROC curves was 0.878 . Selected RQ cutoff value was 1.46 (85\% sensitivity, 83\% specificity). subclinical inflammation by evaluation of molecular phenotype. Gene expression pattern of subclinical and clinical rejection was found to be different. Transcriptional profile of clinical inflammation showed stronger proinflammatory setting compared to subclinical inflammation. Moreover, trend of gradually increasing proinflammatory transcriptome rising from control group, escalating through subclinical and culminating in clinical inflammation group was observed. In other words, subclinical rejection samples showed an intermediate level of inflammatory transcripts in between normal and CI samples.

The quantitative differences seen in transcriptome of SCI and CI are consistent with the theory that subclinical rejection represents an early stage of TCMR and might progress to its clinical phenotype accompanied with graft function deterioration if left untreated. On the contrary, some believe that subclinical infiltration represent a self- limited forme fruste TCMR or injury- repair response to implantation injury rather than true TCMR [21]. However, their observations were based on earlier protocol biopsies performed at 6 weeks posttransplantation when the presence of disturbances in allograft caused by ischemia/ reperfusion injury is more likely than in 3 months

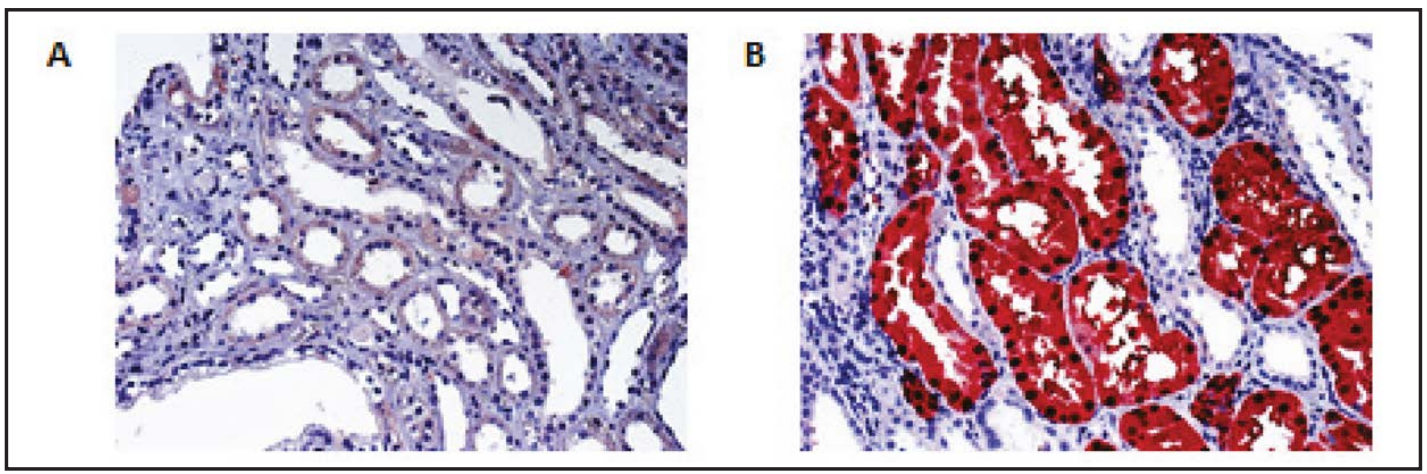

Fig. 3. Immunohistochemical evaluation. Higher intensity of tubular PTGER2 protein staining in nonprogressors (B) compared to progressors (A).

protocol biopsies performed in our study. To exclude that stronger proinflammatory setting of CI in our study is not related to severe grades of rejection, we looked for correlations among the differential gene transcripts and histological inflammation scores and found only weak relationship with tubulitis and interstitial inflammation but no relationship with glomerulitis, intimal arteritis and grade of intragraft inflammation.

Some authors argue that subclinical infiltrates might represent injury-repair response without the functional consequences [21]. The higher incidence of delayed graft function observed in our subclinical inflammation group might be suspected of "injury-repair response" nature of silent T- cell mediated inflammation rather than of the true TCMR. However, no association between differential gene expressions and DGF was found which favoured our decision not to exclude DGF grafts from the analysis. 


\section{Kidney Blood Pressure Research}

Kidney Blood Press Res 2015;40:244-257

\begin{tabular}{l|l}
\hline DOI: $10.1159 / 000368500$ & (C) 2015 S. Karger AG, Basel
\end{tabular}

Published online: May 08, 2015

www.karger.com/kbr

We are aware of fact that borderline changes are not classified by Banff classification as conventional TCMR and their inclusion into studied groups might represent a potential disturbance when interpreting the results of our study. However, subclinical TCMR and borderline changes have showed an identical molecular phenotype [21]. Moreover, some observational studies have shown that persistent inflammation even below the Banff threshold diagnosis for acute rejection, results in worse graft function [29]. Furthermore, the treatment of borderline change is recommended by KDIGO [23]. After all, inter- and intraobserver agreement on the Banff classification grade, especially on borderline lesions, varies significantly among different centres $(4,5)$.

One might argue that the long-term advantage of treatment of subclinical rejection is controversial. However, evidence favours treating subclinical rejection [23]. It is known, that early subclinical inflammation represents a risk factor for the development of interstitial fibrosis $[5,30]$ and persistent inflammation is associated with fibrosis progression and chronic antibody-mediated rejection [31,32]. It was also shown that subclinical antibodymediated rejection is associated with an increased risk of graft loss, irrespective of the kidney allograft function at the time of biopsy [33]. All above mentioned supports the importance of early recognition and surveillance of subclinical inflammation.

We also aimed to validate the applicability of molecular analysis of renal allograft in predicting the graft function deterioration. Some studies suggest that molecular phenotype of protocol biopsies has little relationship to future events and outcomes [21] while the others report findings of potential molecular predictors [22, 34]. In our study, multiple logistic regression analysis revealed that low prostaglandin E2 receptor (PTGER2) gene expression levels were predictive of graft function deterioration or vice versa, higher PTGER2 gene expression level might be associated with protection against progression of renal dysfunction. Prostaglandin E2 (PGE2) is mediating a variety of both innate and adaptive immune responses through 4 distinct receptors: EP1R to EP4R and is widely viewed as a general immunosuppressant [35-37]. It has been reported that PGE2 significantly inhibit allograft rejection in animal transplantation models [38] and exerts its immunosuppressive properties through EP2 and EP4 receptors [39]. EP2R and EP4R agonist might, through the modulation of immune responses, including IFN- $\gamma$ production, inhibition of up-regulation of adhesion and costimulatory molecules represent potential targets for immunosuppressive strategies [40-43]. EP2 receptors directly inhibit T cell proliferation and regulate antigen presenting cells functions [44], inhibit IL-5 and IFN- $\gamma$ production, suggesting suppression both Th1- and Th2- antigen-specific human T-cell responses [45].

Moreover, EP2 and EP4 agonists significantly prolonged cardiac allograft survival in a murine cardiac transplantation model [39]. EP4 agonist was more effective than the EP2 agonist to inhibit acute allograft rejection, suggesting more important role of EP4. Some exciting data have emerged concerning the EP4 selective agonist as a novel agent to protect both posttransplantation inflammatory and alloimmune responses [42, 46-48]. However, in our study we were not able to assess the importance of EP4 receptor in progression of renal dysfunction in kidney allografts with findings of subclinical or clinical inflammation as the PTGER4 gene was not included in the evaluated gene set. Although PGE2 has recently emerged as an immunoactivator through facilitation of Th1 differentiation and Th17 expansion $[49,50]$, the vast majority of studies still report the traditional belief that EP2-EP4 agonists [40,43], not antagonists, can be a suitable therapeutic drug target for inflammation diseases. We believe that PGE2 receptors might merit further study as novel therapeutic for clinical transplantation.

Although we were able to detect higher expression of Treg-specific cell marker (HPGD) in SCI samples, no enhancement of FoxP3 expression was seen. The low expression of FoxP3 in SCI, despite the pure subclinical setting is surprising and contradictory to previous publications, where it was reported as being high, especially in borderline changes [51]. The difference can be explained in part by the inclusion of severe grades of subclinical rejection. 


\section{Kidney \\ Blood Pressure Research}

Kidney Blood Press Res 2015;40:244-257

\begin{tabular}{l|l}
\hline DOI: $10.1159 / 000368500$ & (C) 2015 S. Karger AG, Basel
\end{tabular}

Published online: May 08, 2015

www.karger.com/kbr

However, our study was not designed to show differences in renal allograft transcriptome between different rejection grades so data should not be over interpreted. Decreased expression of KNG1 in CI compared to SCI and control is fully consistent with literature knowledge of decreased kininogen1- proteomic activity in acute cellular rejection [52].

Although protocol biopsies are routinely performed almost in all transplant centres, no uniform opinion on benefit of SCI treatment exists. Furthermore no convincing predictors of graft function deterioration are known. While histological evaluation of renal biopsy remains the current "gold standard" for assessment of acute rejection, molecular analysis might help to understand the background running processes and contribute to elucidate the silent course of subclinical rejection. Some authors believe that histological findings precede functional deterioration. We and others even believe that molecular response precedes histological changes $[53,54]$. Hence, we chose to compare the molecular phenotype of subclinical inflammation in 3 month protocol biopsies with clinical inflammation in early case biopsies. Although the study was limited by the sample size and its descriptive nature, our preliminary findings provide interesting information on transcriptional difference between subclinical and clinical rejections. The degree of alloimmune inflammation gene expression in subclinical inflammation was milder than in clinical inflammation group suggesting the explanation for different clinical course of subclinical rejection

\section{Conclusion}

The presented results might provide additional proof that subclinical acute inflammation has transcriptional profile of acute injury of lower extend than clinical inflammation. This study also supports the concept that rejection is a linear process with the clinically detectable rise in creatinine being the latest event what indirectly emphasizes the importance of early treatment of subclinical rejection to prevent progression to more intense alloimmune activation and development of interstitial fibrosis. If the PTGER2 gene expression is as a potential indicator of graft function deterioration must be validated in further studies.

\section{Abbreviations}

AUC, area under curve; BNIP3L, BH3-only protein family; CASP, caspase; CCL, chemokine ligand; CCR, chemokine receptor; CCRL, chemokine receptor-like; $\mathrm{CD}$, cluster of differentiation; CSF, colony stimulating factor; CXCR, chemokine receptor; CI, clinical inflammation; DGF, delayed graft function; EP, prostaglandin receptor; HPGD, hydroxyprostaglandin dehydrogenase; IL, interleukin; IL1RAPL2, interleukin 1 receptor accessory protein-like 2 ; IFN- $\gamma$, interferon gamma; KDIGO, kidney disease improving global outcomes; KNG1, kinogen-1; LAMP3, lysosome-associated membrane glycoprotein 3; LILRB, leukocyte Iglike receptor; LTA, lymphotoxin A; MAPK 14, mitogen-activated protein kinases; MMF, mycophenolate mofetil; NFKBIE, nuclear factor of kappa light polypeptide gene enhancer in B-cells inhibitor, epsilon; PGE2, prostaglandin E2; PTAFR, platelet-activating factor receptor; PTGER2, prostaglandin E2 receptor; ROC, receiver operating characteristic; RQ, relative quantification; RT-PCR, reverse transcriptase polymerase chain reaction; SCI, subclinical infalmmation; TAC, tacrolimus; TCMR, T-cell mediated rejection; TGFA, transforming growth factor alpha; TGF- $\beta$, transforming growth factor $\beta$; TLDA, Taqman ${ }^{\circledR}$ low density array

\section{Conflict of Interest}

The authors have declared no conflicts of interest. 


\section{Kidney \\ Blood Pressure Research}

\section{Acknowledgements}

The authors thank the transplantation coordinators, nurses and patients for their cooperation and help. Special thanks to Romana Polackova for expert technical assistance and Vera Polaskova for her help with data collection.

O.V. is the recipient of grants from the Internal Grant Agency of the Ministry of Health of the Czech Republic No: NT- 11227-5/2010 and MH CZ DRO ("Institute for Clinical and Experimental Medicine - IKEM, IN 00023001"), the Grant Agency of the Czech Republic (P301/11/1568), Technology Agency CR (TA CR) No: TA03010331,the European Regional Development Fund (ED2.1.00/03.0076), and the 7FP programs of EU BioDrim (7E13020, Ministry of Health, Youth and Sports) and MABSOT.

\section{References}

1 Meier-Kriesche HU, Schold JD, Srinivas TR, Kaplan B: Lack of improvement in renal allograft survival despite a marked decrease in acute rejection rates over the most recent era. Am J Transplant 2004;4:378383.

2 Rush D, Somorjai R, Deslauriers R, Shaw A, Jeffery J, Nickerson P: Subclinical rejection--a potential surrogate marker for chronic rejection--may be diagnosed by protocol biopsy or urine spectroscopy. Ann Transplant 2000;5:44-49.

3 Rush D, Nickerson P, Gough J, McKenna R, Grimm P, Cheang M, Trpkov K, Solez K, Jeffery J: Beneficial effects of treatment of early subclinical rejection: a randomized study. J Am Soc Nephrol 1998;9:2129-2134.

4 Gloor JM, Cohen AJ, Lager DJ, Grande JP, Fidler ME, Velosa JA, Larson TS, Schwab TR, Griffin MD, Prieto M, Nyberg SL, Sterioff S, Kremers WK, Stegall MD: Subclinical rejection in tacrolimus-treated renal transplant recipients. Transplantation 2002;73:1965-1968.

5 Nankivell BJ, Borrows RJ, Fung CL, O'Connell PJ, Allen RD, Chapman JR: Natural history, risk factors, and impact of subclinical rejection in kidney transplantation. Transplantation 2004;78:242-249.

6 El-Amm JM, Gruber SA: The significance of subclinical rejection. Clin Transplant 2009;23:150-156.

7 Masin-Spasovska J, Spasovski G, Dzikova S, Petrusevska G, Lekovski L, Ivanovski N, Popov Z: Do we have to treat subclinical rejections in early protocol renal allograft biopsies? Transplant Proc 2007;39:2550-2553.

8 Kee TY, Chapman JR, O'Connell PJ, Fung CL, Allen RD, Kable K, Vitalone MJ, Nankivell BJ: Treatment of subclinical rejection diagnosed by protocol biopsy of kidney transplants. Transplantation 2006;82:36-42.

9 Nankivell BJ, Chapman JR: The significance of subclinical rejection and the value of protocol biopsies. Am J Transplant 2006;6:2006-2012.

10 Kurtkoti J, Sakhuja V, Sud K, Minz M, Nada R, Kohli HS, Gupta KL, Joshi K, Jha V: The utility of 1- and 3-month protocol biopsies on renal allograft function: a randomized controlled study. Am J Transplant 2008;8:317-323.

11 Scholten EM, Rowshani AT, Cremers S, Bemelman FJ, Eikmans M, van Kan E, Mallat MJ, Florquin S, Surachno J, ten Berge IJ, Bajema IM, de Fijter JW: Untreated rejection in 6-month protocol biopsies is not associated with fibrosis in serial biopsies or with loss of graft function. J Am Soc Nephrol 2006;17:2622-2632.

12 Rush D, Arlen D, Boucher A, Busque S, Cockfield SM, Girardin C, Knoll G, Lachance JG, Landsberg D, Shapiro J, Shoker A, Yilmaz S: Lack of benefit of early protocol biopsies in renal transplant patients receiving TAC and MMF: A randomized study. Am J Transplant 2007;7:2538-2545.

13 Roberts ISD, Reddy S, Russell C, Davies DR, Friend PJ, Handa AI, Morris PJ: Subclinical rejection and borderline changes in early protocol biopsy specimens after renal transplantation. Transplantation 2004;77:1194-1198.

14 Seron D, Diazgallo C, Grino JM, Castelao AM, Carrera M, Bover J, Alsina J: Characterization of Interstitial Infiltrate in Early Renal-Allograft Biopsies in Patients with Stable Renal-Function. Transplant Proc 1991;23:1267-1269.

15 Hoffmann SC, Hale DA, Kleiner DE, Mannon RB, Kampen RL, Jacobson LM, Cendales LC, Swanson SJ, Becker BN, Kirk AD: Functionally significant renal allograft rejection is defined by transcriptional criteria. Am J Transplant 2005;5:573-581. 


\section{Kidney \\ Blood Pressure Research}

Kidney Blood Press Res 2015;40:244-257

\begin{tabular}{l|l}
\hline DOI: $10.1159 / 000368500$ & (c) 2015 S. Karger AG, Basel
\end{tabular}

Published online: May 08, 2015

www.karger.com/kbr

16 Rowshani AT, Florquin S, Bemelman F, Kummer JA, Hack CE, Ten Berge IJ: Hyperexpression of the granzyme B inhibitor PI-9 in human renal allografts: a potential mechanism for stable renal function in patients with subclinical rejection. Kidney Int 2004;66:1417-1422.

17 Grimm PC, McKenna R, Nickerson P, Russell ME, Gough J, Gospodarek E, Liu B, Jeffery J, Rush DN: Clinical rejection is distinguished from subclinical rejection by increased infiltration by a population of activated macrophages. J Am Soc Nephrol 1999;10:1582-1589.

18 Rush DN, Karpinski ME, Nickerson P, Dancea S, Birk P, Jeffery JR: Does subclinical rejection contribute to chronic rejection in renal transplant patients? Clin Transplant 1999;13:441-446.

19 Lipman ML, Shen Y, Jeffery JR, Gough J, McKenna RM, Grimm PC, Rush DN: Immune-activation gene expression in clinically stable renal allograft biopsies: molecular evidence for subclinical rejection. Transplantation 1998;66:1673-1681.

20 Einecke G, Melk A, Ramassar V, Zhu LF, Bleackley RC, Famulski KS, Halloran PF: Expression of CTL associated transcripts precedes the development of tubulitis in T-cell mediated kidney graft rejection. Am J Transplant 2005;5:1827-1836.

21 Mengel M, Chang J, Kayser D, Gwinner W, Schwarz A, Einecke G, Broecker V, Famulski K, de Freitas DG, Guembes-Hidalgo L, Sis B, Haller H, Halloran PF: The molecular phenotype of 6-week protocol biopsies from human renal allografts: reflections of prior injury but not future course. Am J Transplant 2011;11:708-718.

22 Matl I, Hribova, P., Honsova, E., .Brabcova, I., Viklicky, O.: Potential predictive markers in protocol biopsies for premature renal graft loss. Kidney Blood Press Res 2010;33:7-14.

23 KDIGO clinical practice guideline for the care of kidney transplant recipients. Am J Transplant 2009;9 Suppl 3:S1-155.

24 Urbanova M, Brabcova I, Girmanova E, Zelezny F, Viklicky O: Differential regulation of the nuclear factorkappaB pathway by rabbit antithymocyte globulins in kidney transplantation. Transplantation 93:589-596.

25 Sanchez-Espiridion B, Sanchez-Aguilera A, Montalban C, Martin C, Martinez R, Gonzalez-Carrero J, Poderos C, Bellas C, Fresno MF, Morante C, Mestre MJ, Mendez M, Mazorra F, Conde E, Castano A, Sanchez-Godoy P, Tomas JF, Morente MM, Piris MA, Garcia JF: A TaqMan low-density array to predict outcome in advanced Hodgkin's lymphoma using paraffin-embedded samples. Clin Cancer Res 2009;15:1367-1375.

26 Livak KJ, Schmittgen TD: Analysis of relative gene expression data using real-time quantitative PCR and the 2(-Delta Delta C(T)) Method. Methods 2001;25:402-408.

27 Halloran PF, Hunsicker LG: Delayed graft function: state of the art, November 10-11, 2000. Summit meeting, Scottsdale, Arizona, USA. Am J Transplant 2001;1:115-120.

28 Zhang L, Zhao Y: The regulation of Foxp3 expression in regulatory CD4(+)CD25(+)T cells: multiple pathways on the road. J Cell Physiol 2007;211:590-597.

29 Mengel M, Gwinner W, Schwarz A, Bajeski R, Franz I, Brocker V, Becker T, Neipp M, Klempnauer J, Haller H, Kreipe H: Infiltrates in protocol biopsies from renal allografts. Am J Transplant 2007;7:356-365.

30 Heilman RL, Devarapalli Y, Chakkera HA, Mekeel KL, Moss AA, Mulligan DC, Mazur MJ, Hamawi K, Williams JW, Reddy KS: Impact of subclinical inflammation on the development of interstitial fibrosis and tubular atrophy in kidney transplant recipients. Am J Transplant 10:563-570.

31 Moreso F, Carrera M, Goma M, Hueso M, Sellares J, Martorell J, Grinyo JM, Seron D: Early subclinical rejection as a risk factor for late chronic humoral rejection. Transplantation 93:41-46.

32 Wiebe C, Gibson IW, Blydt-Hansen TD, Karpinski M, Ho J, Storsley LJ, Goldberg A, Birk PE, Rush DN, Nickerson PW: Evolution and clinical pathologic correlations of de novo donor-specific HLA antibody post kidney transplant. Am J Transplant 12:1157-1167.

33 Loupy A, Vernerey D, Tinel C, Duong J, Rabant M, Jouven X, Glotz D, Legendre C, Lefaucheur C: Early Recognition of Subclinical Rejection and Outcome of Kidney Allografts. Am J Transplant 2014;14:62-62.

34 Kotsch K, Kunert K, Merk V, Reutzel-Selke A, Pascher A, Fritzsche F, Tullius SG, Pratschke J: Novel markers in zero-hour kidney biopsies indicate graft quality and clinical outcome. Transplantation 90:958-965.

35 van der Pouw Kraan TC, Boeije LC, Smeenk RJ, Wijdenes J, Aarden LA: Prostaglandin-E2 is a potent inhibitor of human interleukin 12 production. J Exp Med 1995;181:775-779.

36 Ferreri NR, Sarr T, Askenase PW, Ruddle NH: Molecular Regulation of Tumor-Necrosis-Factor-Alpha and Lymphotoxin Production in T-Cells - Inhibition by Prostaglandin-E2. J Biol Chem 1992;267:9443-9449. 


\section{Kidney \\ Blood Pressure Research}

Kidney Blood Press Res 2015;40:244-257

\begin{tabular}{l|l}
\hline DOI: $10.1159 / 000368500$ & (c) 2015 S. Karger AG, Basel
\end{tabular}

Published online: May 08, 2015

www.karger.com/kbr

37 Coleman RA, Smith WL, Narumiya S: International Union of Pharmacology Classification of Prostanoid Receptors - Properties, Distribution, and Structure of the Receptors and Their Subtypes. Pharmacol Rev 1994;46:205-229.

38 Kamei T, Callery MP, Flye MW: Intragraft Delivery of 16, 16-Dimethyl Pge2 Induces Donor-Specific Tolerance in Rat Cardiac Allograft Recipients. Transplantation 1991;51:242-246.

39 Nomi T, Sho M, Akahori T, Kanehiro H, Nakajima Y: Protective effect of prostaglandin E2 receptors EP2 and EP4 in alloimmune response in vivo. Transplant Proc 2006;38:3209-3210.

40 Nakase H, Fujiyama Y, Oshitani N, Oga T, Nonomura K, Matsuoka T, Esaki Y, Murayama T, Teramukai S, Chiba T, Narumiya S: Effect of EP4 Agonist (ONO-4819CD) for Patients with Mild to Moderate Ulcerative Colitis Refractory to 5-Aminosalicylates: a Randomized Phase II, Placebo-Controlled Trial. Inflamm Bowel Dis 2010;16:731-733.

41 Takahashi HK, Iwagaki H, Yoshino T, Mori S, Morichika T, Itoh H, Yokoyama M, Kubo S, Kondo E, Akagi T, Tanaka N, Nishibori M: Prostaglandin E(2) inhibits IL-18-induced ICAM-1 and B7.2 expression through EP2/EP4 receptors in human peripheral blood mononuclear cells. J Immunol 2002;168:4446-4454.

42 Morichika T, Takahashi HK, Iwagaki H, Yagi T, Saito S, Kubo S, Yoshino T, Akagi T, Mori S, Nishibori M, Tanaka N: Effect of prostaglandin E2 on intercellular adhesion molecule-1 and B7 expression in mixed lymphocyte reaction. Transplantation 2003;75:2100-2105.

43 Kabashima K, Saji T, Murata T, Nagamachi M, Matsuoka T, Segi E, Tsuboi K, Sugimoto Y, Kobayashi T, Miyachi Y, Ichikawa A, Narumiya S: The prostaglandin receptor EP4 suppresses colitis, mucosal damage and CD4 cell activation in the gut. J Clin Invest 2002;109:883-893.

44 Nataraj C, Thomas DW, Tilley SL, Nguyen MT, Mannon R, Koller BH, Coffman TM: Receptors for prostaglandin E(2) that regulate cellular immune responses in the mouse. J Clin Invest 2001;108:12291235.

45 Okano M, Sugata Y, Fujiwara T, Matsumoto R, Nishibori M, Shimizu K, Maeda M, Kimura Y, Kariya S, Hattori H, Yokoyama M, Kino K, Nishizaki K: E prostanoid 2 (EP2)/EP4-mediated suppression of antigen-specific human T-cell responses by prostaglandin E-2. Immunology 2006;118:343-352.

46 Kuzumoto Y, Sho M, Ikeda N, Hamada K, Mizuno T, Akashi S, Tsurui Y, Kashizuka H, Nomi T, Kubo A, Kanehiro H, Nakajima Y: Significance and therapeutic potential of prostaglandin E-2 receptor in hepatic ischemia/reperfusion injury in mice. Hepatology 2005;42:608-617.

47 Ogawa M, Suzuki J, Kosuge H, Takayama K, Nagai R, Isobe M: The mechanism of anti-inflammatory effects of prostaglandin E2 receptor 4 activation in murine cardiac transplantation. Transplantation 2009;87:16451653.

48 Okamoto T, Okamoto S, Fujimoto Y, Tabata Y, Uemoto S: Suppression of acute rejection by administration of prostaglandin E2 receptor subtype 4 agonist in rat organ transplantation models. J Surg Res 183:852-859.

49 Sakata D, Yao C, Narumiya S: Prostaglandin E2, an immunoactivator. J Pharmacol Sci 112:1-5.

50 Yao CC, Sakata D, Esaki Y, Li YX, Matsuoka T, Kuroiwa K, Sugimoto Y, Narumiya S: Prostaglandin E(2)-EP4 signaling promotes immune inflammation through $\mathrm{T}(\mathrm{H}) 1$ cell differentiation and $\mathrm{T}(\mathrm{H}) 17$ cell expansion. Nature Med 2009;15:633-U141.

51 Nemeth D, Ovens J, Opelz G, Sommerer C, Dohler B, Becker LE, Gross ML, Waldherr R, Mieth M, Sadeghi M, Schmidt J, Langer RM, Zeier M, Susal C: Does borderline kidney allograft rejection always require treatment? Transplantation 90:427-432.

52 Freue GV, Sasaki M, Meredith A, Gunther OP, Bergman A, Takhar M, Mui A, Balshaw RF, Ng RT, Opushneva N, Hollander Z, Li G, Borchers CH, Wilson-McManus J, McManus BM, Keown PA, McMaster WR: Proteomic signatures in plasma during early acute renal allograft rejection. Mol Cell Proteomics 9:1954-1967.

53 Mas VR, Archer KJ, Yanek K, Dumur CL, Capparuccini ML, Mangino MJ, King A, Gibney EM, Fisher R, Posner M, Malur D: Gene expression patterns in deceased donor kidneys developing delayed graft function after kidney transplantation. Transplantation 2008;85:626-635.

54 Wohlfahrtova M, Brabcova I, Zelezny F, Balaz P, Janousek L, Honsova E, Lodererova A, Wohlfahrt P, Viklicky O: Tubular atrophy and low netrin-1 gene expression are associated with delayed kidney allograft function. Transplantation 97:176-183. 